\title{
Neural Systems Underlying the Reappraisal of Personally Craved Foods
}

\author{
Nicole R. Giuliani ${ }^{1}$, Traci Mann ${ }^{2}$, A. Janet Tomiyama ${ }^{3}$, \\ and Elliot T. Berkman ${ }^{1}$
}

\begin{abstract}
Craving of unhealthy food is a common target of selfregulation, but the neural systems underlying this process are understudied. In this study, participants used cognitive reappraisal to regulate their desire to consume idiosyncratically craved or not craved energy-dense foods, and neural activity during regulation was compared with each other and with the activity during passive viewing of energy-dense foods. Regulation of both food types elicited activation in classic top-down selfregulation regions including the dorsolateral prefrontal, inferior frontal, and dorsal anterior cingulate cortices. This main effect of regulation was qualified by an interaction, such that activation in these regions was significantly greater during reappraisal of
\end{abstract}

\section{INTRODUCTION}

Overweight and obesity are at unprecedented levels. As such, gaining a better understanding of the psychological and neural determinants of eating behavior is a pressing topic for research. Food elicits strong appetitive and affective responses that motivate us to consume it (Desmet \& Schifferstein, 2008). In fact, the stimuli themselves do not even need to be present to elicit these responses; pictures of food elicit cravings and other affective responses (Heatherton \& Wagner, 2011; Hofmann, van Koningsbruggen, Stroebe, Ramanathan, \& Aarts, 2010). Advertisers capitalize on these responses, inundating potential customers with pictures of juicy hamburgers, crisp fries, and creamy chocolate desserts. Although some of these advertised foods may be healthy, the billions of advertising dollars spent every year by the fast food industry are mainly focused on marketing unhealthy food (Harris, Schwartz, \& Brownell, 2011). These dollars are not wasted; subjective cravings for energy-dense (ED) foods are associated with an attentional bias toward those foods (Smeets, Roefs, \& Jansen, 2009; Papies, Stroebe, \& Aarts, 2008), increased eating of ED foods (Gilhooly et al., 2007), and future increases in weight (Yokum, Ng, \& Stice, 2011).

\footnotetext{
${ }^{1}$ University of Oregon, ${ }^{2}$ University of Minnesota, ${ }^{3}$ University of California, Los Angeles
}

craved (versus not craved) foods and several regions, including the dorsolateral prefrontal, inferior frontal, medial frontal, and dorsal anterior cingulate cortices, were uniquely active during regulation of personally craved foods. Body mass index significantly negatively correlated with regulation-related activation in the right dorsolateral PFC, thalamus, and bilateral dorsal ACC and with activity in nucleus accumbens during passive viewing of craved (vs. neutral, low-energy density) foods. These results suggest that several of the brain regions involved in the selfregulation of food craving are similar to other kinds of affective self-regulation and that others are sensitive to the self-relevance of the regulation target.

People have the ability to modulate responses to these food stimuli using various forms of self-regulation. One kind of affective self-regulation is cognitive reappraisal, the reinterpretation of an emotional stimulus or event so as to change its meaning (Giuliani \& Gross, 2009). Reappraisal is an effective way of modulating positive emotions in general (Giuliani, McRae, \& Gross, 2008) and craving of preferred foods in particular (Giuliani, Calcott, \& Berkman, 2013). Researchers have proposed that eating behavior is the result of an interplay between appetitive motivation and cognitive control processes such as reappraisal (Nederkoorn, Houben, Hofmann, Roefs, \& Jansen, 2010). A new but substantial body of research has begun to uncover the brain systems involved in desire for and appetitive motivation toward food (e.g., Siep et al., 2009; Pelchat, Johnson, Chan, Valdez, \& Ragland, 2004; Killgore et al., 2003), primarily focusing on obesity (e.g., Carnell, Gibson, Benson, Ochner, \& Geliebter, 2012; De Silva, Salem, Matthews, \& Dhillo, 2012). However, the brain systems involved in regulating those responses in normal weight individuals have received far less attention.

Considerable knowledge about other forms of selfregulation can serve as a starting point for understanding food craving regulation. For example, deficits in selfregulation and executive function suffered by patients with frontotemporal dementia, which include binge eating and strong cravings for sweets and carbohydrates (Mendez, Licht, \& Shapira, 2008), have been traced to 
atrophy of the frontal cortex (Piguet, 2011). Although much of the research on the neuroscience of self-regulation in healthy adults has focused on the regulation of emotion and thought (Heatherton, 2011), work extending these findings to the regulation of a range of targets (e.g., food craving, affect, thought) suggest that, regardless of the regulation target, studies tend to reveal activation during self-regulation in the ventromedial and lateral PFC and the ACC (Heatherton, 2011). A recent review of the brain regions engaged during emotion regulation using cognitive reappraisal found consistent recruitment of lateral PFC and ACC, but not ventromedial PFC (Buhle et al., 2013). Of the two studies to date directly investigating the neural correlates of food craving reappraisal in healthy-weight individuals (Siep et al., 2012; Hollmann et al., 2012), only Hollmann et al. (2012) explicitly compared reappraisal with passive viewing. In that study, reappraisals focusing on the long-term negative consequences of consumption elicited strong activation in PFC (dorsolateral prefrontal and inferior frontal cortices). Furthermore, no studies have examined whether regulation-related brain activity relates to real-world measures associated with food intake, such as body mass index (BMI), in healthy-weight individuals.

In light of the limited number of studies investigating the neural systems of food craving regulation, the present research aims to clarify how the brain supports the self-regulation of food craving using cognitive reappraisal and whether and how activation in those neural systems is related to real-world measures related to food intake. Our study design has two unique features that advance the literature. First, we capitalize on the idiosyncratic nature of food preferences (Rozin \& Vollmecke, 1986) to develop task conditions that enable comparisons that can be difficult to capture with other normed targets of regulation. Although some past studies of the reappraisal of negative emotion have created participant-specific stimuli (e.g., negative autobiographical memories; Goldin et al., 2013), the conditions of the present paradigm allowed us to examine both the general neural systems of food craving regulation (with the main effect of selfregulation vs. viewing) and also the neural systems that are specific to the self-regulation of idiosyncratically craved foods (with the simple effect of self-regulation of craved vs. not craved foods). This second comparison is specifically designed to yield a more ecologically valid picture of how everyday regulation operates because people presumably dedicate more time and effort in their daily lives to regulating targets that are self-relevant (e.g., craved foods, personally emotional stimuli) compared with generic targets that are not necessarily self-relevant (e.g., ED foods that are not craved, emotional images from the International Affective Picture System). Second, we measured BMI as an index of cumulative ED food intake. Weight gain in part results from an imbalance between the energy content of food eaten and the energy expended by the body (Hall et al., 2012; Bolton-Smith \&
Woodward, 1994; George, Tremblay, Depres, LeBlanc, \& Bouchard, 1990), and thus, BMI is expected to be related systematically (although not perfectly) to food intake. We argue that studying the relationship between BMI and neural activation is a logical first step toward understanding how the basic neurocognitive systems involved in cognitive self-regulation are related to behaviors that take place primarily outside the laboratory. As such, this study contributes to an emerging interest within cognitive neuroscience about the links between neural functioning and real-world outcomes (Berkman \& Falk, 2013).

We hypothesize that food craving regulation will elicit brain activation in the domain-general self-regulation and emotion regulation networks reviewed above, including the dorsolateral PFC (DLPFC), inferior frontal gyrus (IFG), and dorsal ACC (dACC). Furthermore, we hypothesize that regulating responses to personally craved ED foods (vs. ED foods that are not personally craved) will recruit additional regions beyond the typical self-regulation network-including the medial PFC, which is implicated in self-relevance processing (Kelley et al., 2002)—but we note that there are no previous results directly relevant to this comparison on which to base a hypothesis. Demonstrating whether or not food craving reappraisal recruits these brain regions is important for several reasons. First, if we can verify that reappraisal of food craving relies upon similar neural regions as reappraisal of other kinds of emotional stimuli, then we could import knowledge gained from studies of emotion regulation into the field of craving regulation. Second, it will broaden the available options for studying reappraisal beyond the standard battery of negative pictures, which are not appropriate for many populations (e.g., children). We also expect that passive viewing of personally craved ED foods (vs. viewing of not craved ED foods) will elicit activation in the nucleus accumbens (NAcc), a hub of the dopaminergic reward system consistently identified in human and animal studies of reward, drug addiction, motivation, and food processing (Demos, Heatherton, \& Kelley, 2012). Lastly, we hypothesize that activation in these regions will relate meaningfully to real-world measures associated with food self-regulation, including BMI and self-reported everyday food regulation. A mechanistic understanding of the selfregulation of appetitive responses to stimuli that are harmful in the long-term has profound implications for both goal-directed food consumption specifically and for cognitive and behavioral self-regulation broadly.

\section{METHODS}

\section{Participants}

Fifty participants ( 17 men, age $M=21.77, S D=2.36$ ), recruited from the University of Oregon undergraduate and graduate student population, completed a study lasting approximately $90 \mathrm{~min}$. Potential participants were excluded if they were left-handed, were below 18 years 
or above 30 years of age, were nonnative English speakers, had a current or past diagnosis of neurological or psychiatric disorder, had a history of head trauma, were pregnant, currently used psychoactive medication, had any non-MRI-compatible conditions (e.g., metal in body), were currently on a diet, were on a diet in the past 3 months, or planned to go on a diet in the next 3 months. To ensure they were neither sated nor food deprived, participants were instructed to eat a full meal approximately $2 \mathrm{hr}$ before the start of the scan. All gave informed consent in accordance with the University of Oregon Institutional Review Board. Two additional participants were excluded from analyses because of a history of brain trauma not disclosed during prescreening.

\section{Task}

Details of the task are outlined in our previous work (Giuliani et al., 2013). Briefly, images of two types of palatable foods were included as stimuli: low-energy density foods ("Neutral") and ED foods of the participants' choosing. The total stimulus set consisted of 20 pictures of low-energy density food (carrots, cauliflower, celery, corn, cucumber, beans, broccoli, Brussels sprouts, eggplant, lettuce, squash, tomatoes; pretested desirability $M=2.48, S D=0.27$ ) and 40 pictures in each of the following categories of ED food (pre-tested desirability $M s=3.46-3.52$, SDs $=0.17-0.35$ ): chocolate, cookies, donuts, fries, ice cream, pasta, and pizza. Importantly, images were chosen such that the mean desirability of the ED food categories were not significantly different from each other (all paired-samples $p>.05$ ) and that the mean of each ED food category was significantly greater than the mean of the Neutral stimuli $(p<.001)$.

All participants saw the same set of 20 Neutral stimuli. For the ED stimuli, participants chose from the list of seven food types listed above the one that they craved the most ("Craved") and the one they craved the least ("Not Craved"), and saw only images within those two categories in addition to the Neutral stimuli during the task. Craving was defined as the desire and tendency to consume the target food, even in the absence of hunger. The ED food trials were organized in a 2 (Stimulus: Craved vs. Not Craved) $\times 2$ (Instruction: Look vs. Regulate) design. The stimuli assigned to each condition were counterbalanced across participants, and the stimuli within each category did not vary from participant to participant. The final event-related design included five trial types (Look Neutral [LN], Look Craved [LC], Look Not Craved [LNC], Regulate Craved [RC], and Regulate Not Craved [RNC]), with 20 trials for each condition totaling 100 trials, distributed across three approximately even runs with brief breaks in between.

The Look instruction directed participants to focus on the pictured food, imagine it was actually in front of them, and think about consuming it. The Regulate instruction directed participants to focus on the food, imagine it was in front of them, and think about it in a way that reduced their desire to eat it. We asked participants to use one of the following four reappraisal strategies specific to food, developed on a separate sample (Giuliani et al., 2013): (1) imagine that you are currently very full, (2) focus on the negative consequences of eating the pictured food (e.g., stomachache, weight gain), (3) remind yourself that you can save the food for later, and (4) imagine that something bad had happened to the food (e.g., sneezed on). Participants chose one strategy before the task and were directed to use that same strategy on every trial. For the rating period, we instructed participants to report their craving honestly at the end of each trial. To minimize the demand characteristics of the task regarding regulation success (i.e., reduced desire ratings on Regulate trials), we explicitly stated "we don't expect you to be able to do this on every picture, so please honestly rate how much you desire the food when all is said and done." To minimize instruction contamination across trials, we instructed participants to view each trial as a fresh event and to do their best to only look or only regulate according to the cue.

Each trial began with a 2-sec instructional cue (Look or Regulate), followed by a 5-sec stimulus presentation, 4 sec to rate the desirability of the stimulus, and a jittered intertrial interval averaging $1 \mathrm{sec}$ and following a gamma distribution. Desirability ratings ("How much do you desire to eat this food?") were made on a 1-to-5 Likert scale, where $1=$ not at all and $5=$ very much. Within-run stimulus order was optimized to maximize contrast estimation efficiency using a genetic algorithm (Wager \& Nichols, 2003). Run order was counterbalanced across participants.

\section{Strategy Training}

Before beginning the task, participants underwent a structured training session in which they received the strategy instructions described above and viewed a sample trial for each of the two instructions. Sample trials provided participants experience with using the cognitive reappraisal strategies while looking directly at pictures of foods not used during the experimental session. The experiment began when the training session was complete, and participants indicated to the experimenter that the directions and procedures were understood.

\section{Experimental Procedure}

After providing informed consent, participants were trained on the task and then asked to choose their most and least craved categories of ED stimuli and the regulation strategy they believed would be most effective 
for them during Regulate trials. Participants then practiced the task with the experimenter (N.G.). In the MRI, participants first completed three runs of the eating regulation task as well as other tasks not reported here. Following the completion of the task, the experimenter interviewed each participant to ensure that they had indeed used the selected regulation strategy on all Regulate trials. Lastly, participants completed the individual difference measures detailed below, reported height, weight, and level of hunger on a 1 (very bungry) to 5 (very full) Likert scale and were thanked for their time.

\section{Behavioral Data Analysis}

Self-reported ratings of desire for the ED foods were subjected to a $2 \times 2$ repeated-measures ANOVA to determine main effects of Stimulus and Instruction and test for interaction effects. Pairwise $t$ tests between conditions were performed to decompose observed effects as well as investigate the differences between LN, LC, and LNC. Regulation success was defined in two ways: (1) percent difference in self-reported desire to consume the pictured food between Regulate and Look (i.e., the main effect of regulation) and (2) the percent difference in self-reported desire between LC to RC (i.e., the simple main effect of regulation of personally-craved foods). Reactivity to food cues was also defined in two ways: (1) percent difference in self-reported desire to consume the pictured food between LN and LC and (2) percent difference in self-reported desire to consume the pictured food between LNC to LC. The alpha level was set to .05 for all analyses. All statistical analyses of behavioral data were performed in SPSS 19.0 (IBM Corp., Armonk, NY).

\section{fMRI Data Acquisition and Analysis}

Data were acquired using a 3.0-T Siemens Allegra headonly scanner at the University of Oregon's Robert and Beverly Lewis Center for Neuroimaging. BOLD-EPI were acquired with a T2*-weighted gradient-echo sequence (repetition time $[\mathrm{TR}]=2000 \mathrm{msec}$, echo time $[\mathrm{TE}]=$ $30 \mathrm{msec}$, flip angle $=80^{\circ}$, matrix size $=64 \times 64,32 \mathrm{con}-$ tiguous axial slices with interleaved acquisition, field of view $=200 \mathrm{~mm}$, slice thickness $=4 \mathrm{~mm}$ ). This sequence also prospectively corrected for motion during acquisition with PACE (Thesen, Heid, Mueller, \& Schad, 2000). For each participant, a high-resolution structural T1-weighted 3-D MP-RAGE pulse sequence $(\mathrm{TR}=2500 \mathrm{msec}, \mathrm{TE}=$ $4.38 \mathrm{msec}$, inversion time $=1100 \mathrm{msec}$, flip angle $=8^{\circ}$, matrix size $=256 \times 192,160$ contiguous axial slices, voxel size $=1 \mathrm{~mm}^{3}$, slice thickness $=1 \mathrm{~mm}$; time 10:49) was acquired coplanar with the functional images. After the functional runs, field map scans were acquired to obtain magnetization values used to correct for field inhomo- geneity $(\mathrm{TR}=500 \mathrm{msec}, \mathrm{TE}=4.99 \mathrm{msec}$, flip angle $=$ $55^{\circ}$, matrix size $=64 \times 64$, field of view $=200 \mathrm{~mm}, 32$ contiguous axial slices with interleaved acquisition, slice thickness $=4 \mathrm{~mm}$ ).

Before preprocessing, nonbrain tissue was removed from the brain images using robust skull stripping with the Brain Extraction Tool in FMRIB's Software Library (FSL; www.fmrib.ox.ac.uk/fsl/). Image preprocessing was implemented in SPM8 (Wellcome Department of Cognitive Neurology, London, UK; www.fil.ion.ucl.ac.uk/ $\mathrm{spm} /$ ), which included correction for field inhomogeneities, realignment, and coregistration of functional images to each subject's own high-resolution structural image using a six-parameter rigid body transformation model, reorientation to the plane containing the anterior and posterior commissures, spatial normalization into space compatible with an MNI atlas, and smoothing using a $6 \mathrm{~mm}^{3}$ FWHM Gaussian kernel. Statistical analyses were implemented in SPM8. For each participant, eventrelated condition effects were estimated according to the general linear model, using a canonical hemodynamic response function, high-pass filtering (128 sec), and a first-order autoregressive error structure. At the individual level, BOLD signal was modeled in a fixed effects analysis with separate regressors modeling each condition of interest during the picture presentation period ( $5 \mathrm{sec}$ ) and for the instruction and rating periods. Linear contrasts were created for each comparison of interest (e.g., RC vs. LC). These contrasts were then imported to group-level random effects analyses for inference to the population.

Because the brain regions previously identified in reappraisal encompass several large cortical and subcortical regions, we investigated the neural correlates of food regulation using whole-brain analyses. For these whole-brain analyses, we applied a combined voxelheight and cluster-extent correction for multiple comparisons to guard against Type I error derived from AFNI's AlphaSim software (Cox, 1996). AlphaSim takes into account the size of the search space and the estimated smoothness of the data (using AFNI's 3dFWHMx) to generate probability estimates (using Monte Carlo simulations) of a random field of noise producing a cluster of voxels of a given size for a set of voxels passing a given voxel-wise $p$-value threshold. In our data set, these simulations determined that a voxel-wise threshold of $p<.001$ combined with a spatial extent threshold of 25 voxels corresponded to a family-wise error (FWE) corrected false-positive probability of $p<$ .05 across the whole brain. For large clusters identified in whole-brain analyses that encompassed multiple anatomical brain areas, we individually interrogated the peaks by creating ROIs. Spherical $(10 \mathrm{~mm})$ ROIs were built in MarsBar (MRC Cognition and Brain Sciences Unit, Cambridge, UK; marsbar.sourceforge.net/), centered at the peak voxel for each anatomically defined region within the larger cluster. 
Although the main focus of this study is food craving regulation, our design also allowed us to replicate the finding that the NAcc is involved in ED food reactivity. Because of the small volume of this region, we created a priori ROIs encompassing the left and right NAcc, as defined by the Harvard-Oxford subcortical atlas in FSL (left: $4280 \mathrm{~mm}^{2}$; right: $4296 \mathrm{~mm}^{2}$ ). These ROIs were then imported into MarsBar, where average ROI signal intensities (beta values) were calculated separately for each condition compared with fixation baseline.

\section{Individual Difference Measures}

We obtained several measures to index the external validity of neural responses during the task. First, we calculated BMI (weight in $\mathrm{kg} /(\text { height in } \mathrm{m})^{2} ; M=$ $21.71, S D=2.8$, range $=17.48-31.63$ ) using selfreported weight and height at the time of scan. Because the distribution of BMI was significantly nonnormal, a squared transformation was applied to improve normality. Second, although self-reported trait measures of regulation do not consistently correlate with task-based laboratory measures (McRae, 2013), we nonetheless administered questionnaires related to food craving regulation that may tap different, more trait-like aspects of food-related self-regulation. Specifically, participants completed the Three-Factor Eating Questionnaire (TFEQ; Stunkard \& Messick, 1985; cognitive restraint average $M=$ $0.44, S D=0.21, \alpha=.82$; disinhibition average $M=0.37$, $S D=0.2, \alpha=.75)$ and the Dutch Eating Behavior Questionnaire (DEBQ; van Strien, Frijters, Bergers, \& Defares, 1986; restrained average $M=2.38, S D=0.83$; $\alpha=$ .92; external average $M=3.33, S D=0.59, \alpha=.8$ ). Relationships between these measures and the beta values extracted from neural activation were calculated using Pearson's correlations in SPSS.

\section{RESULTS}

\section{Behavioral}

Replicating our previous behavioral study with this task (Giuliani et al., 2013), we observed significant main effects of Instruction (Look $M=3.11, S D=0.59$; Regulate $M=$ 2.06, $S D=0.49 ; F(1,49)=106.31, p<.001)$ and Stimulus (Craved $M=3.09, S D=0.48$; Not Craved $M=2.08, S D=$ $0.55 ; F(1,49)=115.05, p<.001)$ on self-reported desire to consume the depicted food. As shown in Figure 1, reappraisal successfully reduced self-reported desire to consume both the Craved $(M=2.44, S D=0.68 ; t(49)=$ $11.02, p<.001)$ and Not Craved foods $(M=1.67, S D=$ $0.46 ; t(49)=7.99, p<.001)$ as compared with passive viewing (Look). This was qualified by a Stimulus $\times$ Instruction interaction $(F(1,49)=38.57, p<.001)$, indicating that the magnitude of regulation success (reduction in selfreported desire) was greater for Craved than Not Craved foods.
Paired samples $t$ tests revealed that reactivity was greater than zero under both definitions (LN to LC, LNC to LC). LC foods $(M=3.75, S D=0.6)$ were rated as significantly more desirable than $\mathrm{LN}(M=2.41, S D=$ $0.84 ; t(49)=10.03, p<.001)$ and LNC foods $(M=2.48$, $S D=0.81 ; t(49)=11.00, p<.001)$, and there was no significant difference in the rated desirability of the LN and LNC stimuli. Neither reactivity definition varied significantly by gender or hunger level.

Across all participants, 21 chose to imagine that something bad had happened to the pictured food (42\%), 17 chose the "focus on the negative consequences of eating that food" strategy (34\%), 11 chose the "imagine that you are currently very full" strategy (22\%), and 1 chose the "remind yourself that you can save that food for later" strategy (2\%). The distribution of participants selecting these strategies is not significantly different from that reported in our previous publication using this paradigm (Giuliani et al., 2013). Regulation success (percent difference in self-reported desire between LC and RC) did not differ significantly by strategy used, gender, or selfreported general hunger of the participant.

\section{fMRI}

\section{Regulation}

As shown in Figure 2A, the main effect of Regulate versus Look across both picture types (Craved, Not Craved) produced a large cluster of activation encompassing the left IFG, anterior insula, and DLPFC, which connected with another cluster in the dACC and pre-SMA. Other clusters included the left-right inferior parietal lobe (IPL) and supramarginal gyrus, right anterior insula, bilateral thalamus, bilateral posterior cingulate (PCC), and right DLPFC (see Table 1). The inverse contrast, the main effect of Look versus Regulate across both picture types, showed

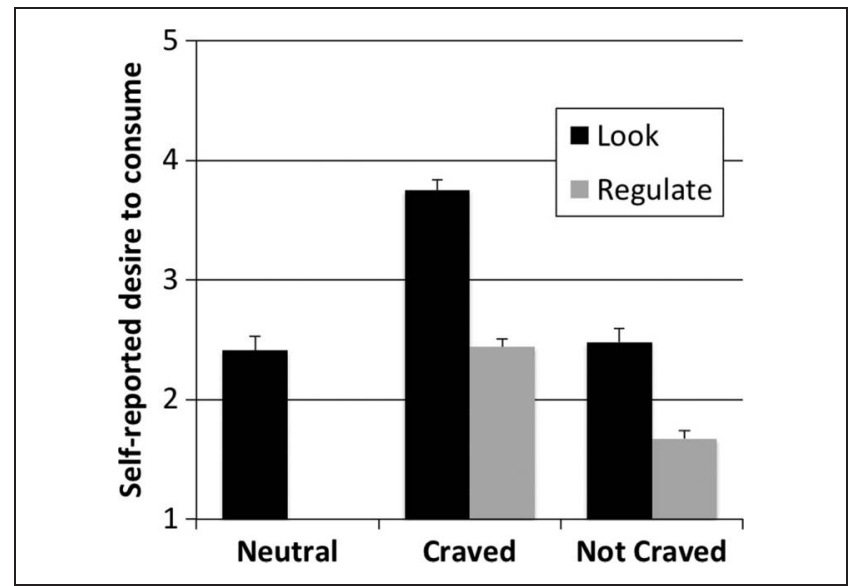

Figure 1. Self-reported mean desire to consume pictured food in the five conditions: look neutral, look craved, look not craved, regulate craved, regulate not craved. Error bars represent SEM. 


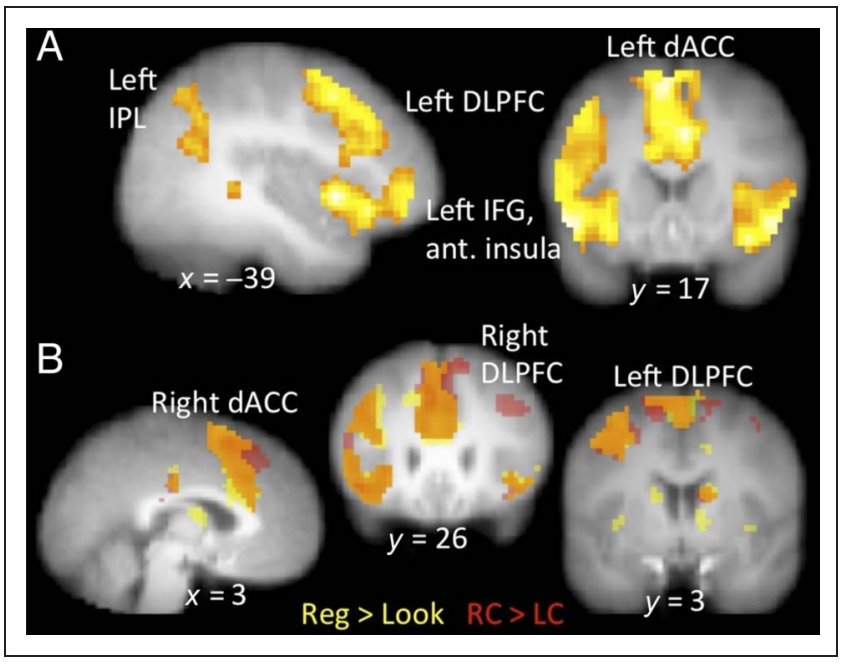

Figure 2. (A) Main effect of Regulate (Regulate Craved + Regulate Not Craved) $>$ Look (Look Craved + Look Not Craved), $p<.001(k=25)$ and (B) a visualization of the overlap of this contrast with Regulate Craved $>$ Look Craved (RC $>$ LC; $p<.001, k=25)$. Voxels only active in the Regulate $>$ Look contrast are in yellow, only RC $>$ LC are in red, and overlapping voxels are in orange.

significant clusters of activation in the bilateral inferior occipital gyri and the left posterior insula.

Further interrogation of the significant clusters from the Regulate > Look contrast broken down by condition produced two distinct activation patterns. First, the clusters located in the bilateral PCC, left and right middle temporal gyrus (MTG) and thalamus, and left IPL and supramarginal gyrus showed the expected pattern of the Regulate $>$ Look main effect in both the Craved and Not Craved conditions. Second, in the large cluster containing left IFG, anterior insula, DLPFC, dACC and pre-SMA, and the smaller clusters located in the right DLPFC and right anterior insula, activation during RC was significantly greater than any of the other four conditions, indicating a possible interaction between Stimulus and Instruction in these regions.

A whole-brain investigation of the voxels significantly more active in the $\mathrm{RC}>\mathrm{LC}$ contrast as compared with the RNC > LNC contrast was conducted to formally test for the Stimulus by Instruction interaction. This contrast revealed additional clusters including the left and right medial superior frontal gyrus (SFG), right DLPFC, and right IPL (Figure 3; for full list see Table 2). To further understand the nature of this interaction, we individually interrogated the simple effects of RC $>$ LC and RC $>$ RNC. The whole-brain contrast of RC $>$ LC (Figure 2B, Table 2) revealed significant clusters of activation in bilateral dACC and pre-SMA, left IFG into anterior insula and DLPFC, left IPL and supramarginal gyrus, right IFG and anterior insula, left MTG, right DLPFC, right superior temporal gyrus, right MTG, right caudate, bilateral PCC,

Table 1. Regions, MNI Coordinates, Cluster Sizes, and Peak $t$ Values for the Regulate $>$ Look and Look $>$ Regulate Main Effects (FWE $p<.05$ Threshold Used for All Contrasts)

\begin{tabular}{|c|c|c|c|}
\hline Contrast and Region & MNI Coordinates $(x, y, z)$ & Cluster Size & Peak $t$ \\
\hline \multicolumn{4}{|c|}{ Regulate $($ Craved + Not Craved $)>$ Look $($ Craved + Not Craved $)$} \\
\hline Left IFG & $(-51,11,-2)$ & 2917 & 8.02 \\
\hline Left anterior insula & $(-30,20,-11)$ & & 7.2 \\
\hline Left DLPFC & $(-36,5,58)$ & & 7.1 \\
\hline Left supramarginal gyrus & $(-51,-49,31)$ & 415 & 7.58 \\
\hline Left IPL & $(-51,-55,43)$ & & 6.87 \\
\hline Right anterior insula & $(42,11,-5)$ & 435 & 7.21 \\
\hline Right cerebellum & $(33,-61,-35)$ & 76 & 7.09 \\
\hline Left middle temporal gyrus & $(-57,-31,-8)$ & 127 & 5.98 \\
\hline Bilateral thalamus & $(0,-7,13)$ & 210 & 5.69 \\
\hline Right middle temporal gyrus & $(48,-31,-11)$ & 105 & 5.49 \\
\hline Bilateral PCC & $(-3,-22,25)$ & 65 & 5.01 \\
\hline Right DLPFC & $(36,47,19)$ & 72 & 4.74 \\
\hline \multicolumn{4}{|c|}{ Look $($ Craved + Not Craved $)>$ Regulate $($ Craved + Not Craved $)$} \\
\hline Right inferior occipital & $(24,-88,-2)$ & 721 & 6.84 \\
\hline Left inferior occipital & $(-18,-94,-8)$ & 284 & 5.84 \\
\hline Left posterior insula & $(-48,-16,16)$ & 44 & 4.24 \\
\hline
\end{tabular}


and right cerebellum. The whole-brain contrast of $\mathrm{RC}>$ RNC (Table 2) revealed significant clusters of activation in the left and right IPL, anterior insula and IFG, bilateral dACC extending into right SFG, bilateral PCC, left middle frontal gyrus (MFG), right DLPFC, and right SFG. None of the activation from these regulation contrasts differed by reappraisal strategy, food category chosen, gender, or hunger.

\section{Reactivity}

Both left and right NAcc were significantly more active during LC compared with LNC (left: $t=2.24, p=.015$; right: $t=1.93, p=.029)$. Interestingly, activation in the left and right NAcc ROIs did not differ significantly between the LC and LN conditions, perhaps because of the heterogeneity of responses to the low-energy density "Neutral" foods. We supplemented these a priori ROI analyses with exploratory whole-brain contrasts investigating both definitions of reactivity (Table 3; all at FWE $p<.05$ ). The contrast of LC $>$ LNC revealed significant clusters in the IPL and lingual gyrus, left IPL and posterior insula, and the bilateral dACC and superior PCC. ${ }^{1}$ The contrast of $\mathrm{LC}>\mathrm{LN}$ revealed three significant clusters of activation, in the right cuneus, precuneus, and parahippocampal gyrus. None of these contrasts varied significantly by food category chosen, gender, or hunger.

\section{Relationships between Neural Activity and Real-world Measures of Regulation and Reactivity}

Regulation

BMI negatively correlated with RC versus LC activity in the thalamus $(r=-.31, p=.032)$ and the right DLFPC $(r=-.29, p=.047$; Figure 4$)$. Within the peaks of the large PFC cluster, the right $(x=6, y=20, z=34 ; r=$ $-.28, p=.049)$ and left dACC $(x=-3, y=23, z=37$; $r=-.28, p=.053)$, BMI, and regulation-related activity were negatively related such that BMI decreased as brain activity during regulation increased. The cognitive restraint subscale of the TFEQ and the restrained subscale of the DEBQ were used to assess the relationship between taskbased neural and self-reported trait indices of food craving regulation. After multiple comparisons correction, regulationrelated brain activity did not relate to either subscale.

\section{Reactivity}

Left and right NAcc ROI activity during reactivity operationalized as LC > LNC did not relate significantly to the disinhibition subscale of the TFEQ, the external subscale of the DEBQ, self-reported reactivity (LC-LN, LCLNC), or to BMI. However, similar to previous work (Demos et al., 2012), NAcc activity during reactivity operationalized as LC > LN positively correlated with BMI

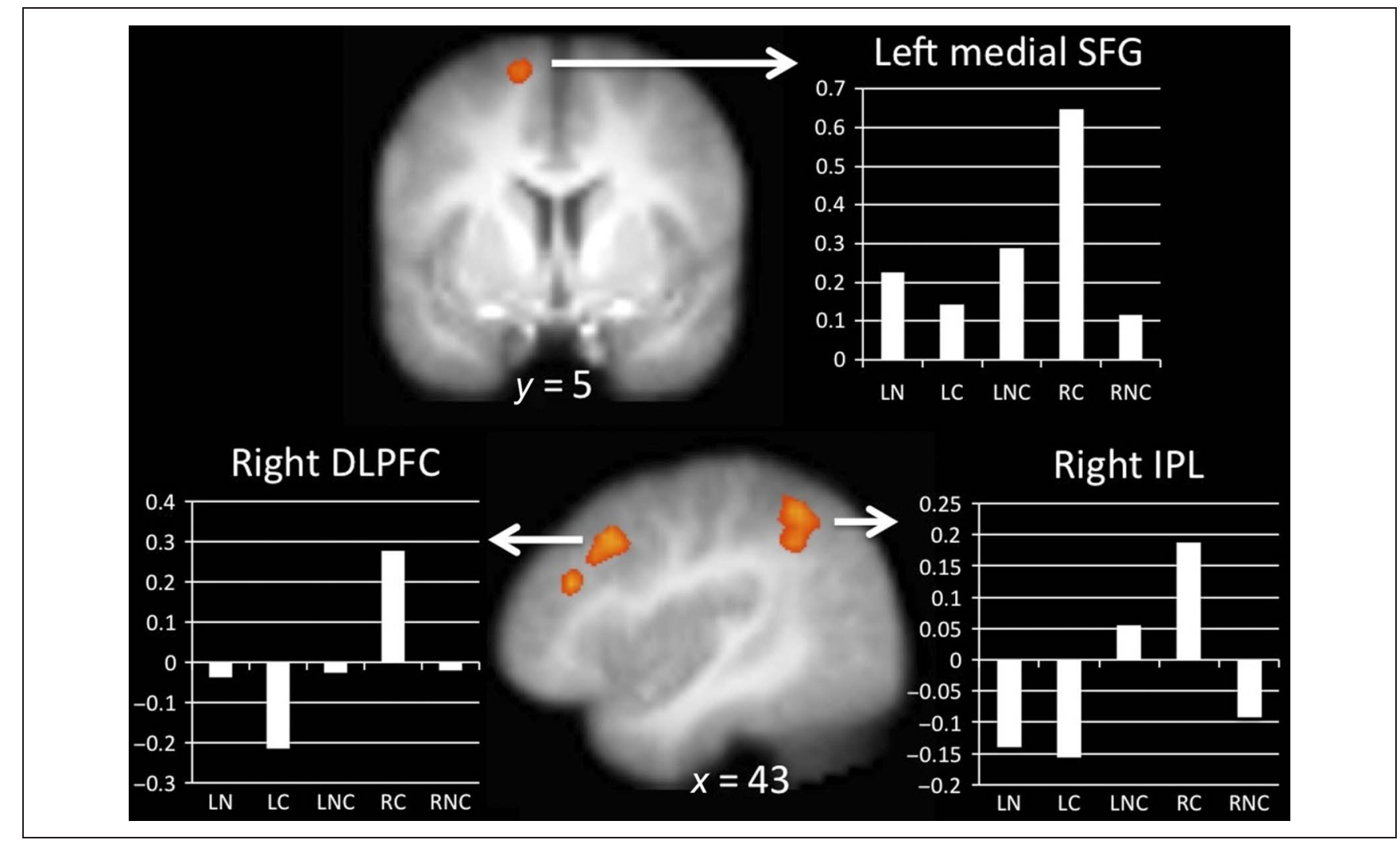

Figure 3. Stimulus $\times$ Instruction interaction $(p<.001, k=25)$ with bar graphs demonstrating the pattern of activation by condition for the left medial SFG, right DLPFC, and right IPL. 
Table 2. Regions, MNI Coordinates, Cluster Sizes, and Peak $t$ Values for the Stimulus $\times$ Instruction, Regulate Craved $>$ Look Craved, and Regulate Craved $>$ Regulate Not Craved Contrasts (FWE $p<.05$ Threshold Used for All Contrasts)

\begin{tabular}{|c|c|c|c|}
\hline Contrast and Region & MNI Coordinates $(x, y, z)$ & Cluster Size & Peak $t$ \\
\hline \multicolumn{4}{|l|}{ Stimulus $\times$ Instruction } \\
\hline Right medial SFG ${ }^{\mathrm{a}, \mathrm{b}}$ & $(6,29,58)$ & 99 & 5.33 \\
\hline Right DLPFC ${ }^{\mathrm{a}, \mathrm{b}}$ & $(39,20,34)$ & 154 & 5.22 \\
\hline Right IPL ${ }^{b}$ & $(39,-52,52)$ & 171 & 5.04 \\
\hline Right precentral gyrus ${ }^{\mathrm{a}, \mathrm{b}}$ & $(33,-4,64)$ & 30 & 4.46 \\
\hline Right precuneus & $(36,-67,43)$ & 38 & 4.41 \\
\hline Left medial SFG ${ }^{\mathrm{a}, \mathrm{b}}$ & $(-12,5,64)$ & 38 & 4.4 \\
\hline \multicolumn{4}{|l|}{ Regulate Craved > Look Craved } \\
\hline Bilateral dACC & $(-3,14,52)$ & 1065 & 8.24 \\
\hline Bilateral SFG & $(0,17,61)$ & & 8.21 \\
\hline Left IFG & $(-45,23,-8)$ & 1435 & 7.82 \\
\hline Left DLPFC & $(-45,2,49)$ & & 7.61 \\
\hline Left IPL & $(-51,-55,43)$ & 405 & 7.41 \\
\hline Left supramarginal gyrus & $(-57,-52,31)$ & & 6.72 \\
\hline Right anterior insula & $(36,11,-11)$ & 463 & 7.2 \\
\hline Right IFG & $(45,20,-11)$ & & 6.2 \\
\hline Right cerebellum & $(33,-58,-35)$ & 78 & 6.79 \\
\hline Left middle temporal gyrus & $(-60,-34,-5)$ & 106 & 5.99 \\
\hline Right DLPFC & $(42,17,43)$ & 240 & 5.17 \\
\hline Right superior temporal gyrus & $(54,-52,34)$ & 69 & 4.71 \\
\hline Right middle temporal gyrus & $(45,-37,-5)$ & 92 & 4.57 \\
\hline Right caudate & $(15,-4,16)$ & 37 & 4.42 \\
\hline Bilateral PCC & $(0,-22,31)$ & 42 & 4.36 \\
\hline \multicolumn{4}{|c|}{ Regulate Craved $>$ Regulate Not Craved } \\
\hline Right IPL & $(39,-43,49)$ & 278 & 5.77 \\
\hline Bilateral dACC into right SFG & $(6,29,58)$ & 573 & 5.41 \\
\hline Right anterior insula & $(42,20,1)$ & 289 & 4.96 \\
\hline Right IFG & $(57,14,7)$ & & 4.9 \\
\hline Right DLPFC & $(42,20,43)$ & 126 & 4.91 \\
\hline Left MFG (pre-SMA) & $(-39,-4,49)$ & 94 & 4.81 \\
\hline Left anterior insula & $(-36,17,4)$ & 54 & 4.64 \\
\hline Left IFG & $(-42,20,-11)$ & & 3.76 \\
\hline Right SFG & $(12,50,40)$ & 28 & 4.52 \\
\hline Bilateral PCC & $(3,-22,31)$ & 45 & 4.39 \\
\hline Left IPL & $(-33,-49,46)$ & 47 & 4.16 \\
\hline
\end{tabular}

${ }^{a}$ Regions from the Stimulus $\times$ Instruction interaction that also showed a significant difference in the RC $>$ LC simple contrast.

${ }^{\mathrm{b}}$ Regions from the Stimulus $\times$ Instruction interaction that also showed a significant difference in the RC $>$ RNC simple contrast. 
Table 3. Regions, MNI Coordinates, Cluster Sizes, and Peak $t$ Values for the Look Craved $>$ Look Neutral and Look Craved $>$ Look Not Craved Contrasts (FWE $p<.05$ Threshold Used for All Contrasts)

\begin{tabular}{llc}
\hline Contrast and Region & Coordinates $(x, y, z)$ & Cluster Size \\
\hline Look Craved $>$ Look Neutral & $(15,-94,7)$ & 46 \\
Right cuneus & $(27,-64,43)$ & 92 \\
Right precuneus & $(36,-76,16)$ & 25 \\
Right middle occipital gyrus & $(27,-46,-20)$ & 37 \\
Right parahippocampal gyrus & & 4.82 \\
& $(54,-37,31)$ & 3.97 \\
Look Craved $>$ Look Not Craved & $(12,-70,-5)$ & 47 \\
Right IPL & $(3,11,31)$ & 42 \\
Right lingual gyrus & $(-48,-22,19)$ & 48 \\
Bilateral dACC & $(3,-4,46)$ & 4.88 \\
Left IPL/posterior insula & 92 \\
Bilateral superior PCC & 4.54 \\
\hline
\end{tabular}

across all participants (left: $r=.33, p=.019$; right: $r=$ $.43, p=.002)$.

A follow-up analysis of the relationships between reactivity- and regulation-related brain activity and BMI showed that regulation-related right DLPFC activation moderated the relationship between reactivity-related right NAcc activation and $\mathrm{BMI}(t(1,46)=3.3, p=.076)$ such that the positive relationship between BMI and right NAcc activity during reactivity was attenuated with increased right DLPFC activity during regulation.

\section{DISCUSSION}

This study examined how self-regulation of craving for tempting foods influenced self-reported desire and neural

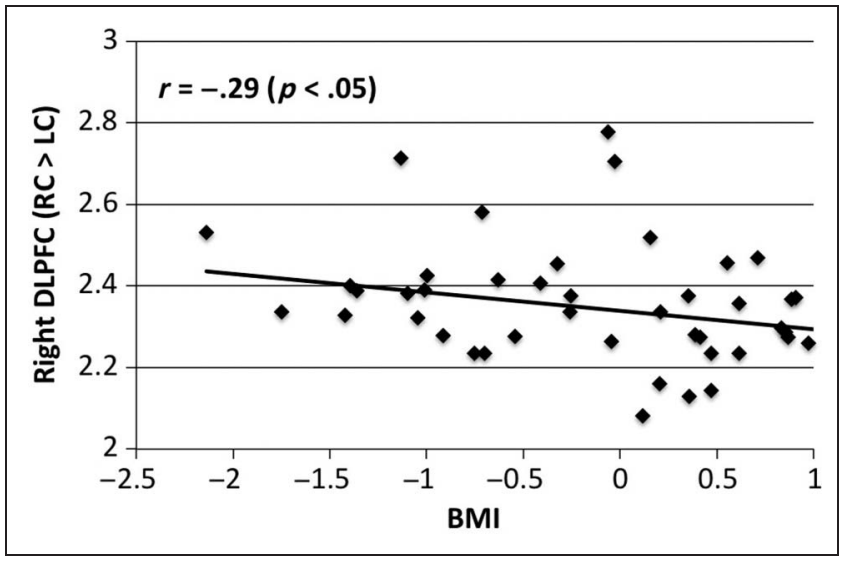

Figure 4. Scatterplot demonstrating the inverse relationship between Regulate Craved > Look Craved ( $\mathrm{RC}>\mathrm{LC}$ ) activation in the right DLPFC (peak at $42,17,43$; square root transformation to improve normality) and BMI (square transformation and $z$-scored). activation. We capitalized on the idiosyncratic nature of food craving to characterize the brain systems specific to regulating personally craved foods as well as those involved in regulating appetitive desires in general. Reappraisal effectively decreased self-reported desire for food and elicited activation in the left IFG and IPL, left and right DLPFC, anterior insula, and MTG, and bilateral thalamus, dACC, and PCC. Activation in a subset of those regions (DLPFC, IFG, and dACC) was specific to regulation of personally craved foods. Finally, regulation-related activity in the thalamus, bilateral dACC, and right DLPFC was correlated with BMI, which is related to overall ED food intake (Hall et al., 2012; Bolton-Smith \& Woodward, 1994; George et al., 1990).

For both self-reported reduction in food craving and reappraisal-related brain activation, there was a difference between regulation of idiosyncratically craved and not craved foods. Self-report ratings revealed a significant stimulus type (Craved, Not Craved) by instruction (Look, Regulate) interaction such that the regulation-related decrease in food desire was greater for the Craved versus Not Craved foods. Within the brain regions showing significantly more activity during regulation of ED foods versus passive viewing, some appeared to be regulationgeneral (e.g., similar levels of activation during both RC and RNC as compared with LC and LNC) whereas others showed significantly greater activation during RC trials compared with any other trial type. A direct comparison investigation of the stimulus by instruction interaction revealed that the left and right medial SFG, right DLPFC, IPL precentral gyrus, and precuneus displayed significantly more activation during the regulation of craving for Craved foods ( $\mathrm{RC}>\mathrm{LC}$ ) compared with the regulation of craving for Not Craved foods ( RNC > LNC). Within these regions, the left and right medial DLPFC, 
right SFG, and right precentral gyrus were recruited by regulation of both targets but to a greater degree for the reappraisal of personally craved foods, whereas the right IPL was uniquely recruited during craved food regulation. This supports our hypothesis that reappraising personally craved foods recruits the medial PFC regions implicated in the processing of self-relevant information (Kelley et al., 2002). Furthermore, it indicates that reappraisal of personally relevant appetitive stimuli recruits an overlapping but distinct set of brain regions than general craving regulation and suggests that regulation of idiosyncratic stimuli may be different than regulation of generic stimuli. The burgeoning field of food craving regulation has recognized the importance of using idiosyncratic stimuli (e.g., Hollmann et al., 2012), but studies of other types of self-regulation (e.g., emotion regulation) are just beginning to use this approach (e.g., Goldin et al., 2013). This emerging body of literature suggests that taking the self-relevance of the target into account is critical for future research on self-regulation. Indeed, it may be the case that the self-relevance of stimuli is a robust moderator of other effects studied in cognitive neuroscience more broadly. If so, the field could capitalize on self-relevance by taking advantage of current advances in computer and information technology that allow scientists to build highly individualized simulations of real-world phenomena in the lab.

Interestingly, the brain regions classically recruited by cognitive reappraisal of negative emotional stimuli (Buhle et al., 2013), including the dACC and DLPFC, were among those recruited to a greater degree by reappraisal of personally craved foods. In other words, the data presented here clearly implicate these regions in the selfregulation of appetitive motives (e.g., craving). Taken together with previous data, this provides good evidence of the flexibility of those regions to regulate both approach and avoidance (e.g., fear, disgust) responses. Although the activation in these regions was seen in the main effect, simple effect analyses revealed that they were driven by RC-related activation. We believe that these stimuli recruit these brain regions because of the high amount of conflict resolution (dACC) and top-down regulation (DLPFC, IFG) required to reappraise craved foods as compared with not craved foods. These findings have strong implications for food consumption interventions aimed at reducing the intake of ED foods, as individuals who find this sort of direct top-down cognitive regulation of desire to be challenging may be directed to find other regulation strategies (e.g., avoiding the foods via situation selection or modification; Gross, 1998).

Reappraisal of both craved and not craved foods significantly reduced activation in the left and right occipital cortex and the left posterior insula (Look $>$ Regulate). Although it is surprising that we did not find ventral striatum (e.g., NAcc) down-regulation during reappraisal, this null result is not unprecedented. For example, Hollmann et al. (2012) found no reduction in ventral striatum activity during regulation; in fact, they found increased dorsal striatum activity during the cognitive control of eating, which they interpreted to reflect increased integration of reward and goal-directed behavior. The relatively few extant fMRI studies on the cognitive control of food craving make it challenging to know exactly what role the striatum plays in this process. One possibility is that null findings may be because of the relatively small anatomical size of the dorsal and ventral striatum. Another possibility, which warrants further study after two independent null findings, is that the field may need to reconsider the role of the ventral striatum in food craving regulation. It may be that ventral striatum responses to food cues are relatively immune to reappraisal and that decreased reports of craving result solely from increased regulatory activity "overpowering" the reactivity response without decreasing it.

We observed increased activation in the occipital lobe and NAcc during the viewing of craved foods, which replicates previous work on food cue reactivity (Demos et al., 2012; Schur et al., 2009). Perhaps not surprisingly, the patterns of reactivity to craved foods differed, depending on the comparison condition. Only the comparison of LC to LNC stimuli produced significantly greater activation in the NAcc, but this activation was not related to self-reported food cravings or weight. In contrast, comparing BOLD signal during viewing of craved foods to healthy (neutral) stimuli did not elicit significant activation in the NAcc, but the degree of activation NAcc in this contrast was significantly related to BMI. This relationship supports previous findings in the field, which documented the positive correlation between NAcc activation and food stimuli (Born et al., 2011), as well as actual snack consumption (Lawrence, Hinton, Parkinson, \& Lawrence, 2012). These results indicate that both neutral and personally undesired ED foods are useful control conditions because they may contain distinct information.

Given the high level of heterogeneity in eating behavior and food-related self-regulation across people, the dualrelationship between BMI with both regulation- and reactivity-related brain activation is of particular interest. First, although they were both correlated with BMI, regulation (DLPFC) and reactivity (NAcc) activity were not significantly related to each other. This suggests that there may be at least two separable pathways through which food-related neural responses are related to cumulative food consumption measures such as BMI. Second, an exploratory analysis of these relationships showed that the positive relationship between BMI and right NAcC activity during reactivity was attenuated with increased right DLPFC activity during regulation. Although the interpretability of this finding is limited by the trend-level significance of this finding and the self-reported nature of the height and weight values used to calculate BMI, this indicates that regulation-related activation of the DLPFC may be of particular interest to investigators developing 
targeted interventions, as it moderates the link between neural reactivity to craved food stimuli and BMI.

Although brain activity during regulation and reactivity related meaningfully to $\mathrm{BMI}$, we also did not see a relationship between task-based neural or behavioral indices of food regulation and self-reported trait food regulation as measured by the TFEQ and DEBQ. This uncoupling of regulation frequency and success has been documented in the literature (see McRae, 2013), and the present findings add to a growing body of research observing this effect. The present findings also challenge the restrictionbased nature of current dieting interventions, which ask people to eat less ED food but do not generally teach them how to mange their cravings for these popular foods. We suggest that affecting the antecedent mental processes associated with the consumption of ED foods via reappraisal as may be more successful than simply targeting the consumption behavior itself. Furthermore, although BMI was used as a proxy for cumulative ED food intake and related significantly to both food reactivity- and regulation-related brain activity, more sensitive measures of body composition and eating behavior may produce more sensitive indices of these brain-behavior relationships. Third, although previous research has noted marked gender differences in eating behavior and body weight regulation (Cornier, Salzberg, Endly, Bessesen, \& Tregellas, 2010; Wang et al., 2009; Rolls, Federoff, \& Guthrie, 1991), we did not find any significant gender effects in this study. This may be because we selectively recruited participants who were not actively dieting, thereby removing known gender effects on food conflict and dieting (Rolls et al., 1991). Lastly, personally craved and not craved foods were identified at the category level, but not at the actual stimulus level. We accepted this limitation to equate the categories on normative ratings, but it is possible that the observed differences between craved and not craved foods would have been even more pronounced if participants were able to identify each image as idiosyncratically craved or not.

In this study, we showed that reappraisal of ED food stimuli recruits top-down control brain regions recruited by other forms of self-regulation. For many of these regions, including the DLPFC and dACC, this recruitment was driven by the reappraisal of idiosyncratically craved foods, perhaps because of the greater cognitive control and conflict resolution required to successfully reduce the desire to consume these foods. The knowledge that these two regions are heavily recruited during this cognitive process may help us in the future to leverage interventions that we know target the functioning of these regions through training (see Schweizer, Grahn, Hampshire, Mobbs, \& Dalgleish, 2013) and apply them to eating. Furthermore, the present results have strong implications for stimulus choice in self-regulation paradigms, as they demonstrate that the self-relevance of stimuli can significantly affect both the magnitude and pattern of associated neural activity. In other words, self-relevance may be a robust moderator not only of self-regulation of food craving but also of other effects studied in cognitive neuroscience. As such, self-relevance may be a doubleedged sword: on one hand, as a nuisance variable, the degree to which an individual relates to a stimulus may artificially inflate correlations between neural responses to that stimulus and other variables like self-report; on the other hand, scientists may be able to boost the power of small $\mathrm{N}$ studies by leveraging the increased effect sizes that might be associated with tailored stimuli. The present findings answer calls for a greater breadth of experimental paradigms to study emotion regulation generally (Carter, 2009) and also test the range of the flexibility of the neural circuits involved therein to target different motivational and affective responses. Both reactivity-related activation in the NAcc and reappraisalrelated activation in the DLPFC, thalamus, and dACC significantly related to BMI, suggesting that real-world patterns of food consumption, which influence body weight, may result from both reactivity to and regulation of food cues. Indeed, the present findings indicate that a neurally informed intervention that targets both systems may indeed be the most effective means of reducing the consumption of ED food.

\section{Acknowledgments}

We would like to thank the Lewis Center for Neuroimaging, Kyle Burger, and the members of the Developmental Social Neuroscience and Social Affective Neuroscience Laboratories for support and helpful comments on this experimental design and manuscript.

Reprint requests should be sent to Nicole R. Giuliani, 1227 University of Oregon, Eugene, OR 97403-1227, or via e-mail: giuliani@ uoregon.edu.

\section{Note}

1. Unsurprisingly, relaxing the cluster threshold reveals a small cluster in the left NAcc $(t=3.33 ; k=2$; peak at $x=-12$, $y=17, z=-8)$, an anatomical region small enough that we did not expect it to survive the twenty-five $3-\mathrm{mm}^{3}$ voxel cluster threshold.

\section{REFERENCES}

Berkman, E. T., \& Falk, E. B. (2013). Beyond brain mapping: Using neural measures to predict real-world outcomes. Current Directions in Psychological Science, 22, 45-50.

Bolton-Smith, C., \& Woodward, M. (1994). Dietary composition and fat to sugar ratios in relation to obesity. International Journal of Obesity, 18, 820-828.

Born, J. M., Lemmens, S. G., Martens, M. J., Formisano, E., Goebel, R., \& Westerterp-Plantenga, M. S. (2011). Differences between liking and wanting signals in the human brain and relations with cognitive dietary restraint and body mass index. American Journal of Clinical Nutrition, 94, 392-403.

Buhle, J. T., Silvers, J. A., Wager, T. D., Lopez, R., Onyemekwu, C., Kober, H., et al. (2013). Cognitive reappraisal of emotion: 
A meta-analysis of human neuroimaging studies. Cerebral Cortex. Epub ahead of print.

Carnell, S., Gibson, C., Benson, L., Ochner, C. N., \& Geliebter, A. (2012). Neuroimaging and obesity: Current knowledge and future directions. Obesity Reviews, 13, 43-56.

Carter, C. S. (2009). The ups and downs of emotion regulation. Biological Psychiatry, 65, 359-360.

Cornier, M. A., Salzberg, A. K., Endly, D. C., Bessesen, D. H., \& Tregellas, J. R. (2010). Sex-based differences in the behavioral and neuronal responses to food. Physiology \& Behavior, 99, 538-543.

Cox, R. W. (1996). AFNI: Software analysis and visualization of functional magnetic resonance neuroimages. Computers and Biomedical Research, 29, 162-173.

De Silva, A., Salem, V., Matthews, P. M., \& Dhillo, W. S. (2012). The use of functional MRI to study appetite control in the CNS. Experimental Diabetes Research, 2012, Article 764017

Demos, K. E., Heatherton, T. F., \& Kelley, W. M. (2012). Individual differences in nucleus accumbens activity to food and sexual images predict weight gain and sexual behavior. Journal of Neuroscience, 32, 5549-5552.

Desmet, P. M. A., \& Schifferstein, H. N. J. (2008). Sources of positive and negative emotions in food experience. Appetite, 50, 290-301.

George, V., Tremblay, A., Depres, J. P., LeBlanc, C., \& Bouchard, C. (1990). Effects of dietary fat content on total and regional adiposity in men and women. International Journal of Obesity, 14, 1085-1091.

Gilhooly, C. H., Das, S. K., Golden, J. K., McCrory, M. A., Dallal, G. E., Saltzman, E., et al. (2007). Food cravings and energy regulation: The characteristics of craved foods and their relationship with eating behaviors and weight change during 6 months of dietary energy restriction. International Journal of Obesity, 31, 1849-1858.

Giuliani, N. R., Calcott, R. D., \& Berkman, E. T. (2013). Piece of cake. Cognitive reappraisal of food craving. Appetite, 64, 56-61.

Giuliani, N. R., \& Gross, J. J. (2009). Reappraisal. In D. Sander \& K. R. Scherer (Eds.), Oxford companion to the affective sciences (pp. 329-330). New York: Oxford University Press.

Giuliani, N. R., McRae, K., \& Gross, J. J. (2008). The up- and down-regulation of amusement: Experiential, behavioral and autonomic consequences. Emotion, 8, 714-719.

Goldin, P. R., Ziv, M., Jazaieri, H., Hahn, K., Heimberg, R., \& Gross, J. J. (2013). Impact of cognitive behavioral therapy for social anxiety disorder on the neural dynamics of cognitive reappraisal of negative self-beliefs: Randomized clinical trial JAMA Psychiatry, 70, 1048-1056.

Gross, J. J. (1998). Antecedent- and response-focused emotion regulation: Divergent consequences for experience, expression, and physiology. Journal of Personality and Social Psychology, 74, 224-237.

Hall, K. D., Heymsfield, S. B., Kemnitz, J. W., Klein, S., Schoeller, D. A., \& Speakman, J. R. (2012). Energy balance and its components: Implications for body weight regulation. The American Journal of Clinical Nutrition, 95, 989-994.

Harris, J. L., Schwartz, M. B., \& Brownell, K. D. (2011). Fast food FACTS: Evaluating fast food nutrition and marketing to youth. New Haven, CT: Rudd Center for Food Policy and Obesity, Yale University.

Heatherton, T. F. (2011). Neuroscience of self and selfregulation. Annual Review of Psychology, 62, 363-390.

Heatherton, T. F., \& Wagner, D. D. (2011). Cognitive neuroscience of self-regulation failure. Trends in Cognitive Science, 15, 132-139.

Hofmann, W., van Koningsbruggen, G. M., Stroebe, W., Ramanathan, S., \& Aarts, H. (2010). As pleasure unfolds: Hedonice responses to tempting food. Psychological Science, 21, 1863-1870.
Hollmann, M., Hellrung, L., Pleger, B., Schlogl, H., Kabisch, S., Stumvoll, M., et al. (2012). Neural correlates of the volitional regulation of the desire for food. International Journal of Obesity, 36, 648-655.

Kelley, W. M., Macrae, C. N., Wyland, C. L., Caglar, S., Inati, S., \& Heatherton, T. F. (2002). Finding the self? An event-related fMRI study. Journal of Cognitive Neuroscience, 14, 785-794.

Killgore, W. D., Young, A. D., Femia, L. A., Bogorodzki, P., Rogowska, J., \& Yurgelun-Todd, D. A. (2003). Cortical and limbic activation during viewing of high- versus low-calorie foods. Neuroimage, 19, 1381-1394.

Lawrence, N. S., Hinton, E. C., Parkinson, J. A., \& Lawrence, A. D. (2012). Nucleus accumbens response to food cues predicts subsequent snack consumption in women and increased body mass index in those with reduced selfcontrol. Neuroimage, 63, 415-422.

McRae, K. (2013). Emotion regulation frequency and success: Separating constructs from methods and time scale. Social and Personality Psychology Compass, 7, 289-302.

Mendez, M. F., Licht, E. A., \& Shapira, J. S. (2008). Changes in dietary or eating behavior in frontotemporal dementia versus Alzheimer's disease. American Journal of Alzheimer's Disease and Other Dementias, 23, 280-285.

Nederkoorn, C., Houben, K., Hofmann, W., Roefs, A., \& Jansen, A. (2010). Control yourself or just eat what you like? Weight gain over a year is predicted by an interactive effect of response inhibition and implicit preference for snack foods. Health Psychology, 29, 389-393.

Papies, E. K., Stroebe, W., \& Aarts, H. (2008). The allure of forbidden food: On the role of attention in self-regulation. Journal of Experimental Social Psychology, 44, 1283-1292.

Pelchat, M. L., Johnson, A., Chan, R., Valdez, J., \& Ragland, J. D. (2004). Images of desire: Food-craving activation during fMRI. Neuroimage, 23, 1486-1493.

Piguet, O. (2011). Eating disturbance in behavioural-variant frontotemporal dementia. Journal of Molecular Neuroscience, 45, 589-593.

Rolls, B. J., Federoff, I. C., \& Guthrie, J. F. (1991). Gender differences in eating behavior and body weight regulation. Health Psychology, 10, 133-142.

Rozin, P., \& Vollmecke, T. A. (1986). Food likes and dislikes. Annual Review of Nutrition, 6, 433-456.

Schur, E. A., Kleinhans, N. M., Goldberg, J., Buchwald, D., Schwartz, M. W., \& Maravilla, K. (2009). Activation in brain energy regulation and reward centers by food cues with choice of visual stimulus. International Journal of Obesity, 33, 653-661.

Schweizer, S., Grahn, J., Hampshire, A., Mobbs, D., \& Dalgleish, T. (2013). Training the emotional brain: Improving affective control through emotional working memory training. Journal of Neuroscience, 33, 5301-5311.

Siep, N., Roefs, A., Roebroeck, A., Havermans, R., Bonte, M. L., \& Jansen, A. (2009). Hunger is the best spice: An fMRI study of the effects of attention, hunger and calorie content on food reward processing in the amygdala and orbitofrontal cortex. Behavioural Brain Research, 198, 149-158.

Siep, N., Roefs, A., Roebroeck, A., Havermans, R., Bonte, M. L., \& Jansen, A. (2012). Fighting food temptations: The modulating effects of short-term cognitive reappraisal, suppression, and up-regulation on mesocorticolimbic activity related to appetitive motivation. Neuroimage, 60, 213-220.

Smeets, E., Roefs, A., \& Jansen, A. (2009). Experimentally induced chocolate craving leads to an attentional bias in increased distraction but not in speeded detection. Appetite, 53, 370-375.

Stunkard, A. J., \& Messick, S. (1985). The Three-Factor Eating Questionnaire to measure dietary restraint, 
disinhibition, and hunger. Journal of Psychosomatic Research, 29, 71-83.

Thesen, S., Heid, O., Mueller, E., \& Schad, L. R. (2000). Prospective acquisition correction for head motion with image-based tracking for real-time fMRI. Magnetic Resonance in Medicine, 44, 457-465.

van Strien, T., Frijters, J. E. R., Bergers, G. P. A., \& Defares, P. B. (1986). The Dutch Eating Behavior Questionnaire (DEBQ) for assessment of restrained, emotional, and external eating behavior. International Journal of Eating Disorders, 5, 295-315.
Wager, T. D., \& Nichols, T. E. (2003). Optimization of experimental design in fMRI: A general framework using a genetic algorithm. Neuroimage, 18, 293-309. Wang, G. J., Volkow, N. D., Telang, F., Jayne, M., Ma, Y., Pradhan, Z., et al. (2009). Evidence of gender differences in the ability to inhibit brain activation elicited by food stimulation. Proceedings of the National Academy of Sciences, U.S.A., 106, 1249-1254.

Yokum, S., Ng, J., \& Stice, E. (2011). Attentional bias to food images associated with elevated weight and future weight gain: An fMRI study. Obesity, 19, 1775-1783. 